\title{
A Riemannian Framework for Orientation Distribution Function Computing
}

\author{
Jian Cheng ${ }^{1,2}$, Aurobrata Ghosh ${ }^{2}$, Tianzi Jiang ${ }^{1}$, and Rachid Deriche ${ }^{2}$ \\ ${ }^{1}$ LIAMA Research Center for Computational Medicine, Institute of Automation, Chinese \\ Academy of Sciences, China \\ 2 Odyssée Project Team, INRIA Sophia Antipolis - Méditerranée, France \\ \{Jian.Cheng, Aurobrata. Ghosh\}@sophia.inria.fr
}

\begin{abstract}
Compared with Diffusion Tensor Imaging (DTI), High Angular Resolution Imaging (HARDI) can better explore the complex microstructure of white matter. Orientation Distribution Function (ODF) is used to describe the probability of the fiber direction. Fisher information metric has been constructed for probability density family in Information Geometry theory and it has been successfully applied for tensor computing in DTI. In this paper, we present a state of the art Riemannian framework for ODF computing based on Information Geometry and sparse representation of orthonormal bases. In this Riemannian framework, the exponential map, logarithmic map and geodesic have closed forms. And the weighted Frechet mean exists uniquely on this manifold. We also propose a novel scalar measurement, named Geometric Anisotropy (GA), which is the Riemannian geodesic distance between the ODF and the isotropic ODF. The Renyi entropy $H_{\frac{1}{2}}$ of the ODF can be computed from the GA. Moreover, we present an Affine-Euclidean framework and a Log-Euclidean framework so that we can work in an Euclidean space. As an application, Lagrange interpolation on ODF field is proposed based on weighted Frechet mean. We validate our methods on synthetic and real data experiments. Compared with existing Riemannian frameworks on ODF, our framework is model-free. The estimation of the parameters, i.e. Riemannian coordinates, is robust and linear. Moreover it should be noted that our theoretical results can be used for any probability density function (PDF) under an orthonormal basis representation.
\end{abstract}

\section{Introduction}

Diffusion Tensor Imaging (DTI) [1], based on the assumption of Gaussian diffusion, is a popular way to probe the white matter in vivo. Riemannian frameworks (Affine Invariant framework and Log-Euclidean framework) have been proposed for computing on tensor valued data [2]3/4]5]. These frameworks have proved useful for regularizing, registering, segmenting, and interpolating tensor data. The Riemannian framework provides the intrinsic way to deal with objects in a non-Euclidean space. High Angular Resolution Diffusion Imaging (HARDI) is used to probe non-Gaussian diffusion which represents more intricate microstructure in the tissue. In HARDI data modeling the role of the Orientation Distribution Function (ODF) [6] is pivotal. It is an antipodally symmetric probability density function defined on $S^{2}$ that is sufficiently smooth to be represented by an orthonormal basis, such as the High Order Tensor (HOT) basis [7],

G.-Z. Yang et al. (Eds.): MICCAI 2009, Part I, LNCS 5761, pp. 911-918 2009.

(C) Springer-Verlag Berlin Heidelberg 2009 
the Spherical Harmonic (SH) basis [8], or the Spherical Wavelet basis [9], using only a few coefficients.

A Riemannian framework has never been proposed to our knowledge for the orthonormal basis representation of ODFs. In [6], the author used Kullback-Leibler divergence, however, it's not a real metric. In [10], ODFs were represented in the SH basis and the authors used the $L 2$ distance between the coefficient vectors as the distance between ODFs. The $L 2$ distance is computationally efficient but it's an ambient distance, not a geodesic. Apart from orthonormal bases, special function mixture models have also been proposed to represent ODFs [11/12]. In the case of the von Mises-Fisher distributions, the Riemannian framework can be constructed on the mixture model [13]. However, not every ODF can be represented by these functions, as the von Mises-Fisher distributions don't form a basis. Additionally, there is no efficient method for estimating the parameters and the authors used an iterative optimization approach. Also, there is no natural way to define a metric in a multiplicative space because different spaces should have different weighted coefficients, but the authors considered the same weights in all the spaces.

In this paper, we propose a natural, computationally efficient and model-free Riemannian framework for computing on ODFs. First we study the parameter space of ODFs based on the orthonormal basis representation, and propose the geodesic, the exponential map, the logarithmic map, the weighted Frechet mean, and the Geometric Anisotropy (GA) and the Renyi entropy (RE). The GA we propose is the Riemannian geodesic to a spherical ODF, it indicates how non-spherical an ODF is. Next we also propose the Affine-Euclidean (AE) and the Log-Euclidean (LE) frameworks to be able to work in an Euclidean space. Finally we propose an interpolation method and computation of the mean ODF of a set of ODFs as example applications, based on weighted Frechet mean, and demonstrate our method on synthetic and real data.

\section{Riemannian Framework for ODF}

ODFs have been successfully represented in orthonormal bases. Compared with the special function mixture model in [11/12], basis representations are model-free methods because linear combination of these bases can represent all ODFs. In this section, we will construct the Riemannian framework under the orthonormal basis representation.

\subsection{Parametric Family and Riemannian Framework}

The ODF is a PDF denoted by $p(x), x \in \chi, \chi=S^{2}$. Let the square-root of the ODF be denoted by $\sqrt{p}: x \rightarrow \sqrt{p(x)}, \forall x \in \chi$. We assume a very weak and reasonable assumption that the square-root $\sqrt{p}$ can be represented by the linear combination of the first $K$ bases, i.e. $\sqrt{p}(x)=\sum_{i=1}^{K} c_{i} B_{i}(x) .\left\{B_{i}(x)\right\}_{i \in N}$ is any orthonormal basis function set, and $c=\left(c_{1}, c_{2}, \ldots, c_{K}\right)^{T}$ is called the Riemannian Coordinate. Thus $c$ is the parameter of ODF $p(x \mid c)$, and we have the probability family $P F_{K}$ as

$$
P F_{K}=\left\{p(x \mid \boldsymbol{c})=\left(\sum_{i=1}^{K} c_{i} B_{i}(x)\right)^{2}: \int_{\chi} p(x \mid c) d x=\sum_{i=1}^{K} c_{i}^{2}=1, \sum_{i=1}^{K} c_{i} B_{i}(x) \geq 0, \forall x \in \chi\right\}
$$



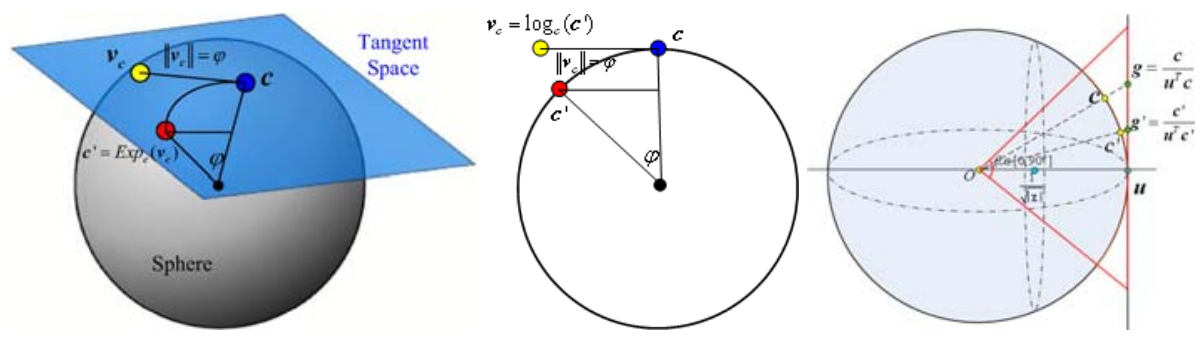

Fig. 1. Left $\mathcal{G}$ Middle: Riemannian framework. Right: Parameter space, Affine-Euclidean and $\log$-Euclidean frameworks. The isotropic ODF $\boldsymbol{u}$ is only marked as an example and may not be at the center of the cone.

All ODFs will be in $P F_{K}$ if $K$ is large enough thanks to the orthonormal basis representation. Let $\partial_{i}=\frac{\partial}{\partial \boldsymbol{c}_{i}}$, then we have the information metric as [13]:

$$
\begin{aligned}
g_{i j}(\boldsymbol{c}) & =\int_{\chi} \partial_{i} \log (p(x \mid \boldsymbol{c})) \partial_{j} \log (p(x \mid \boldsymbol{c})) p(x \mid \boldsymbol{c}) d x=4 \int_{\chi} \partial_{i} \sqrt{p(x \mid \boldsymbol{c})} \partial_{j} \sqrt{p(x \mid \boldsymbol{c})} d x \\
& =4 \int_{\chi} \partial_{i}\left(\sum_{m=1}^{K} c_{m} B_{m}(x)\right) \partial_{j}\left(\sum_{n=1}^{K} c_{n} B_{n}(x)\right) d x=4 \int_{\chi} B_{i}(x) B_{j}(x) d x=4 \delta_{i j}
\end{aligned}
$$

We do not care about a constant multiplier in a metric if we want to devise a computational method. So we consider $g_{i j}=\delta_{i j}$. From the formulae above, we can find that the parameter space $P S_{K}=\left\{\boldsymbol{c} \mid\|\boldsymbol{c}\|=\sum_{i=1}^{K} c_{i}^{2}=1, \sum_{i=1}^{K} c_{i} B_{i}(x) \geq 0, \forall x \in \chi\right\}$ is just a subset of the sphere $S^{k-1}$. The sphere is a simple manifold which has been well studied. Thus we can get the geodesic (the part of the great circle on the sphere), exponential map, and logarithmic map very easily [1411|15]. If we let $\sqrt{p}(x \mid c)=\sum_{i=1}^{K} c_{i} B_{i}(x)$, $\sqrt{p}\left(x \mid \boldsymbol{c}^{\prime}\right)=\sum_{j=1}^{K} c_{j}^{\prime} B_{j}(x)$, and $\boldsymbol{v}_{\boldsymbol{c}}$ is the tangent vector at $\boldsymbol{c}$ towards $\boldsymbol{c}^{\prime}$, then we have the Riemannian framework (see Fig-1):

$$
\begin{gathered}
\text { Distance }: d_{g_{i j}}\left(p(\cdot \mid \boldsymbol{c}), p\left(\cdot \mid \boldsymbol{c}^{\prime}\right)\right)=d_{\delta_{i j}}\left(\boldsymbol{c}, \boldsymbol{c}^{\prime}\right)=\arccos \left(\sum_{i=1}^{K} c_{i} c_{i}^{\prime}\right) \\
\operatorname{Exp}: \operatorname{Exp}_{\boldsymbol{c}}\left(\boldsymbol{v}_{\boldsymbol{c}}\right)=\boldsymbol{c}^{\prime}=\boldsymbol{c} \cos \varphi+\frac{\boldsymbol{v}_{\boldsymbol{c}}}{\left\|\boldsymbol{v}_{\boldsymbol{c}}\right\|} \sin \varphi, \text { where } \varphi=\left\|\boldsymbol{v}_{\boldsymbol{c}}\right\| \\
\log : \log _{\boldsymbol{c}}\left(\boldsymbol{c}^{\prime}\right)=\boldsymbol{v}_{\boldsymbol{c}}=\frac{\boldsymbol{c}^{\prime}-\boldsymbol{c} \cos \varphi}{\left\|\boldsymbol{c}^{\prime}-\boldsymbol{c} \cos \varphi\right\|} \varphi, \text { where } \varphi=\arccos \left(\sum_{i=1}^{K} c_{i} c_{i}^{\prime}\right)
\end{gathered}
$$

The geodesic between ODF $p(x \mid \boldsymbol{c})$ and ODF $p\left(x \mid \boldsymbol{c}^{\prime}\right)$ is $\gamma(t): p(x \mid \boldsymbol{c}(t))$, where $\boldsymbol{c}(t)=$ $\operatorname{Exp}_{\boldsymbol{c}}\left(t \log _{\boldsymbol{c}}\left(\boldsymbol{c}^{\prime}\right)\right)$. Then $\gamma(0)=\boldsymbol{c},\left.\frac{d \gamma(t)}{d t}\right|_{t=0}=\boldsymbol{v}_{\boldsymbol{c}}, \gamma(1)=\boldsymbol{c}^{\prime}$. Actually, it's the part of the great circle that connects $c$ and $c^{\prime}$.

\subsection{Parameter Space, Affine-Euclidean and Log-Euclidean Frameworks}

To use the Riemannian framework, we see from Equ-1 that we should keep the parameter $c$ in $P S_{K}$. To do so we derive useful theoretical results on $P S_{K}$. 
Property-1: $P S_{K}$ is closed. The boundary of $P S_{K}$ is $\left\{c \mid \sum_{i=1}^{K} c_{i}^{2}=1, \exists C \subset \chi\right.$, s.t. $x \in$ $\left.C, \sum_{i=1}^{K} c_{i} B_{i}(x)=0, \forall x \notin C, \sum_{i=1}^{K} c_{i} B_{i}(x)>0\right\}$. Therefore, $P S_{K}$ is closed by definition. Property-2: $P S_{K}$ is a convex subset of sphere $S^{K-1}$. If $c \in P S_{K}$ and $c^{\prime} \in P S_{K}$, let $\boldsymbol{c}(t), t \in[0,1]$ be the geodesic between them. Now, $\forall t \in[0,1], \exists \lambda \in[0,1]$, s.t. $\boldsymbol{c}(t)=$ $\frac{\lambda \boldsymbol{c}+(1-\lambda) \boldsymbol{c}^{\prime}}{\left\|\lambda \boldsymbol{c}+(1-\lambda) \boldsymbol{c}^{\prime}\right\|}$, because $\boldsymbol{c}(t)$ is on the great circle (geodesic) and $\lambda \boldsymbol{c}+(1-\lambda) \boldsymbol{c}^{\prime}$ is on the chord between $\boldsymbol{c}$ and $\boldsymbol{c}^{\prime}$. And $p(x \mid \boldsymbol{c}(t))=\sum_{i=1}^{K} c_{i}(t) B(x)=\frac{\sum_{i=1}^{K}\left(\lambda \sqrt{p}(x \mid \boldsymbol{c})+(1-\lambda) p\left(x \mid \boldsymbol{c}^{\prime}\right)\right)}{\left\|\lambda \boldsymbol{c}+(1-\lambda) \boldsymbol{c}^{\prime}\right\|} \geq 0$.

Property-3: $P S_{K}$ is contained in a convex cone with angle $90^{\circ} . \forall \mathbf{c}, \mathbf{c}^{\prime} \in P S_{K}$, $\int_{\chi} \sqrt{p}(x \mid \mathbf{c}) \sqrt{p}\left(x \mid \mathbf{c}^{\prime}\right) d x=\int_{\chi} \sum_{i=1}^{K} c_{i} B_{i}(x) \sum_{j=1}^{K} c_{j} B_{j}(x) d x=\sum_{i=1}^{K} c_{i} c_{i}^{\prime}=\cos \left(\mathbf{c}, \mathbf{c}^{\prime}\right) \in[0,1]$. That means the angle between any two points in $P S_{K}$ is less than $90^{\circ}$ (see Fig-1).

Property-4: The projection of any $c \in P S_{K}$ on the Riemannian Coordinate $\mathbf{u}$ of the isotropic ODF should be more than $\frac{1}{\sqrt{4 \pi}}$. If the isotropic ODF is denoted by $U(x)=$ $\frac{1}{|\chi|}=\frac{1}{4 \pi}$, and $\sqrt{U}(x)=\frac{1}{\sqrt{|x|}}=\sum_{i=1}^{K} u_{i} B_{i}(x)$, then $\forall \boldsymbol{c} \in P S_{K}, \boldsymbol{c}^{T} \boldsymbol{u}=\cos (\boldsymbol{c}, \boldsymbol{u})=$ $\int_{\chi} \sqrt{p}(x \mid c) \frac{1}{\sqrt{|\chi|}}>\frac{1}{\sqrt{|\chi|}} \int_{\chi} p(x \mid c) d x=\frac{1}{\sqrt{4 \pi}}$. That means the projection of $\boldsymbol{c}$ on $\boldsymbol{u}$ should be more than $\frac{1}{\sqrt{4 \pi}}$. If we choose the SH basis, $\forall \boldsymbol{c}=\left(c_{1}, c_{2}, \ldots, c_{K}\right) \in P S_{K}, c_{1}>\frac{1}{\sqrt{4 \pi}}$ because $\boldsymbol{u}=(1,0, \ldots, 0)$.

Result-1: Geometric Anisotropy (GA). Thanks to property-2, in order to measure the anisotropy of an ODF, it is possible to define GA, a scalar function, as the geodesic distance between the ODF $p(x \mid c)$ and the isotropic ODF $U(x)$, i.e.

$$
G A(p(x \mid \boldsymbol{c}))=d(p(\cdot \mid \boldsymbol{c}), U(\cdot))=\arccos \left(\boldsymbol{c}^{T} \boldsymbol{u}\right) \in\left[0, \arccos \left(\frac{1}{\sqrt{4 \pi}}\right)\right)
$$

Result-2: Renyi Entropy (RE). The RE of order $\frac{1}{2}$ is

$$
\begin{aligned}
H_{\frac{1}{2}}(p(x \mid \boldsymbol{c})) & =2 \log \left(\int_{\chi} \sqrt{p}(x \mid \boldsymbol{c}) d x\right)=2 \log (\cos (G A(p(x \mid \boldsymbol{c}))))+\log (4 \pi) \\
& =\log \left(4 \pi\left(\boldsymbol{c}^{T} \boldsymbol{u}\right)^{2}\right) \in(0, \log (4 \pi)]
\end{aligned}
$$

Result-3: Affine-Euclidean $(A E) \mathcal{E}$ Log-Euclidean $(L E)$ frameworks. Since $P S_{K}$ is a closed and convex subset of a hemisphere including $\boldsymbol{u}$ (properties-1,2,3,4), it is diffeomorphic to a subset, named $E u c$, of the tangent space of $\boldsymbol{u}$. We can define a diffeomorphism $F: P S_{K} \subset S^{K-1} \rightarrow E u c \subset T_{\boldsymbol{u}}$. We can use the metric in Euc to redefine the metric in $P S_{K}$. Then the Riemannian framework will be changed so that we can work in an Euclidean space, which will approximate the Riemannian framework (see Fig-1). If we Let $F_{*}$ and $F^{*}$ denote respectively the push forward and pull back maps, then

$$
\begin{gathered}
\text { Distance : } d_{P S_{K}}\left(p(\cdot \mid \boldsymbol{c}), p\left(\cdot \mid \boldsymbol{c}^{\prime}\right)\right)=d_{E u c}\left(F(\boldsymbol{c}), F\left(\boldsymbol{c}^{\prime}\right)\right)=\left\|F(\boldsymbol{c})-F\left(\boldsymbol{c}^{\prime}\right)\right\| \\
\operatorname{Exp}: \operatorname{Exp} \boldsymbol{c}\left(\boldsymbol{v}_{\boldsymbol{c}}\right)=\boldsymbol{c}^{\prime}=F^{-1}\left(F(\boldsymbol{c})+F_{*} \boldsymbol{v}_{\boldsymbol{c}}\right) \\
\log : \log _{\boldsymbol{c}}\left(\boldsymbol{c}^{\prime}\right)=\boldsymbol{v}_{\boldsymbol{c}}=F^{*}\left(F\left(\boldsymbol{c}^{\prime}\right)-F(\boldsymbol{c})\right)
\end{gathered}
$$

If we choose $F(\boldsymbol{c})=\log _{\boldsymbol{u}}(\boldsymbol{c})$, then we get the Log-Euclidean framework like the one in tensor space [4]. If we choose $F(\boldsymbol{c})=\frac{\boldsymbol{c}}{\boldsymbol{u}^{T} \boldsymbol{c}}-\boldsymbol{u}$, we get the Affine-Euclidean framework, which is very popular in Statistical Shape Analysis (SSA) [5 16]. 


\subsection{Weighted Frechet Mean}

Another important property of $P S_{K}$ is that the weighted Frechet mean exists uniquely [14]. The weighted Frechet mean is defined as the point on the manifold $P S_{K}$ that minimizes the summation of weighted squared Riemannian geodesic distances to each sample point [3]. If $f_{1}, f_{2}, \ldots, f_{N} \in P S_{K}, Q_{\boldsymbol{w}}(f)=\sum_{i=1}^{N} w_{i} d\left(f, f_{i}\right)^{2}, \boldsymbol{w}=\left(w_{1}, \ldots, w_{N}\right)^{T}, w_{i} \in$ $[0,1], \sum_{i=1}^{N} w_{i}=1$, then

$$
\mu_{\boldsymbol{w}}=\arg \min _{f \in P S_{K}} Q_{\boldsymbol{w}}(f)=\arg \min _{f \in P S_{K}} \sum_{i=1}^{N} w_{i} d\left(f, f_{i}\right)^{2}
$$

$Q_{\boldsymbol{w}}\left(\mu_{\boldsymbol{w}}\right)$ is called as sample weighted variance. In [14], the author proved that Frechet mean on hemisphere exists uniquely, so it does in our parameter space because $\boldsymbol{c}^{T} \boldsymbol{u}>$ $\frac{1}{\sqrt{4 \pi}}$. Borrowing from the notation in [14], we denote $\mu_{\boldsymbol{w}} \triangleq \widetilde{\sum}_{i=1}^{N} w_{i} f_{i} \triangleq w_{1} f_{1} \oplus w_{2} f_{2} \oplus$ $\cdots \oplus w_{N} f_{N}$. Here is a gradient descent method to calculate the weighted Frechet mean.

\section{Algorithm 1. Weighted Frechet Mean}

Input: $f_{1}, \ldots, f_{N} \in P S^{K}, \boldsymbol{w}=\left(w_{1}, \ldots, w_{N}\right)^{\prime}, w_{i} \geq 0, i=1,2, \ldots, N, \sum_{i=1}^{N} w_{i}=1$.

Output: $\mu_{\boldsymbol{w}}$, the Weighted Frechet Mean.

$$
\begin{aligned}
& \text { Initialization: } \mu_{\boldsymbol{w}}^{(0)}=\frac{\sum_{i=1}^{N} w_{i} f_{i}}{\left\|\sum_{i=1}^{N} w_{i} f_{i}\right\|}, k=0 \\
& \text { Do } \\
& \quad v_{\mu_{\boldsymbol{w}}^{(k)}}=\sum_{i=1}^{N} w_{i} \log _{\mu_{\boldsymbol{w}}^{(k)}}\left(f_{i}\right) \\
& \mu_{\boldsymbol{w}}^{(k+1)}=\operatorname{Exp}_{\mu_{\boldsymbol{w}}^{(k)}}\left(v_{\mu_{\boldsymbol{w}}^{(k)}}\right) \\
& k=k+1
\end{aligned}
$$

while $\left\|v_{\mu_{w}^{(k)}}\right\|>\varepsilon$

For AE and LE frameworks, because we use the distance on Euclidean spaces to approximate the distance on $P S$, obviously there is a closed form:

$$
\mu_{\boldsymbol{w}}=F^{-1}\left(\sum_{i=1}^{N} w_{i} F\left(f_{i}\right)\right)
$$

\section{Riemannian Coordinate Estimation and ODF Interpolation}

In the previous section, our theoretical results are independent of the choice of the basis function, and we assume that for every ODF $p(x \mid c)$, we know the Riemannian Coordinate $c$. Here we show a simple and linear method to estimate it from raw HARDI data under the $\mathrm{SH}$ basis representation.

First, we can use any method to estimate the ODF $p(x)$. Here we choose the robust, linear, analytical Q-ball imaging [8] with the regularization term. Second, we take discrete samples $p\left(x_{i}\right)$ of the ODF on the continuous sphere, and calculate the squareroot of these samples $\sqrt{p\left(x_{i}\right)}$. Third, we use these square-root samples to estimate the square-root of the ODF through a least squares SH basis fit linearly without regularization. If we choose the first $N$ order SH bases in the first step $(N \geq 4)$ [8], and the first $M$ order in the third step, we should keep $M \geq N$. The advantages of our estimation method are its linearity, robustness and speed, thanks to the analytical Q-ball imaging. 
Based on the weighted Frechet mean in section 2.3, we propose a Lagrange interpolation method on ODF space as an application. In 1-dimension case, if we have $N$ ODFs $\left\{f\left(x_{i}\right), i=1,2, \ldots, N\right\}$ in $N$ spatial position $\left\{x_{i}, i=1,2, \ldots, N\right\}$., we can interpolate the ODF in the whole spatial axis as the weighted Frechet mean of $\left\{f\left(x_{i}\right)\right\}$, i.e. $f(x)=\widetilde{\sum}_{i=1}^{N} w_{i}(x) f\left(x_{i}\right), w_{i}(x)=\prod_{l=1, l \neq i}^{N} \frac{x-x_{l}}{x_{i}-x_{l}}$. It should be noted that if $N=2$, then $f(x)=(1-t) f\left(x_{1}\right) \oplus t f\left(x_{2}\right), t=\frac{x-x_{1}}{x_{2}-x_{1}}$. Actually, in that case, $f(x)$ is the geodesic determined by $f\left(x_{1}\right)$ and $f\left(x_{2}\right)$, i.e. $f(x)=\operatorname{Exp}_{f\left(x_{1}\right)}\left(t \log _{f\left(x_{1}\right)}\left(f\left(x_{2}\right)\right)\right)$. For HARDI image in 3-dimension, if we have $N_{1}, N_{2}$ and $N_{3}$ for ODFs for each dimension, i.e. $\left\{f\left(x_{i}, y_{j}, z_{k}\right), 1 \leq i \leq N_{1}, 1 \leq j \leq N_{2}, 1 \leq k \leq N_{3}\right\}$, we can interpolate ODF in the continuous 3D space as the weighted Frechet mean of $\left\{f\left(x_{i}, y_{j}, z_{k}\right)\right\}$.

$$
\begin{gathered}
f(x, y, z)=\widetilde{\sum}_{i=1}^{N_{1}} \widetilde{\Sigma}_{j=1}^{N_{2}} \widetilde{\Sigma}_{k=1}^{N_{3}} w_{i}^{(1)}(x) w_{j}^{(2)}(y) w_{k}^{(3)}(z) f\left(x_{i}, y_{j}, z_{k}\right) \\
w_{i}^{(1)}(x)=\prod_{l=1, l \neq i}^{N_{1}} \frac{x-x_{l}}{x_{i}-x_{l}}, w_{j}^{(2)}(y)=\prod_{m=1, m \neq j}^{N_{2}} \frac{y-y_{m}}{y_{j}-y_{m}}, w_{k}^{(3)}(z)=\prod_{n=1, n \neq k}^{N_{3}} \frac{z-z_{n}}{z_{k}-z_{n}}
\end{gathered}
$$

\section{Experiments and Results}

Experiments were conducted to illustrate the proposed metrics. Synthetic ODFs were generated using the multi-tensor model ( 1 or 2 fibers, every tensor with eigenvalues $[1700,300,300] \times 10^{-6} \mathrm{~mm}^{2} / \mathrm{s}$ ) [17]. Riemannian Coordinates of ODFs were estimated using the method proposed in section-3. In the experiments, we chose order 4 to estimate the ODF and its square-root with satisfactory results. Then the Lagrange interpolation method was applied to interpolate the ODFs in 1D or 2D cases.

GA was calculated for synthetic data and clinical real data, and compared with GFA [6]. No normalization was used for ODF visualization. Since RE $H_{\frac{1}{2}} \in(0, \log (4 \pi)]$, we plotted its normalized version $H_{\frac{1}{2}} / \log (4 \pi)$. From the results, we find that the three proposed metrics - AE, LE, Riemannian (RM), give very similar results. The RE is close to 1 . This is because the ODF proposed in [6] is very smooth and close to the isotropic ODF. The results of the experiments-A to $\mathrm{E}$ are given in the Fig-2

Exp-A: We interpolate from the isotropic ODF $U(x)$ to a single fiber using the (1)AE, (2) LE, (3) RM metrics. As expected, the GA is linear, since the GA is the Riemannian distance to $\boldsymbol{u}$. Exp-B: We illustrate Riemannian geodesic interpolation between different fiber configurations, and plot the corresponding GA. Exp-C: 2D Lagrange interpolation using the (1)AE, (2) LE, (3) RM metrics. The background is the GA. Exps-A, B, C: ODFs coloured by GA. Exp-D: GA (left) from clinical real data compared to GFA (right). ODFs estimated using [8]. Exp-E: Mean ODF computation from a field of ODFs using the (1) LE and (2) RM metrics.

\section{Conclusion}

In this paper, we proposed a novel Riemannian framework based on Information Geometry and orthonormal basis representation. Our framework is model-free and computationally efficient. The estimation method of Riemannian Coordinates is fast, linear and 

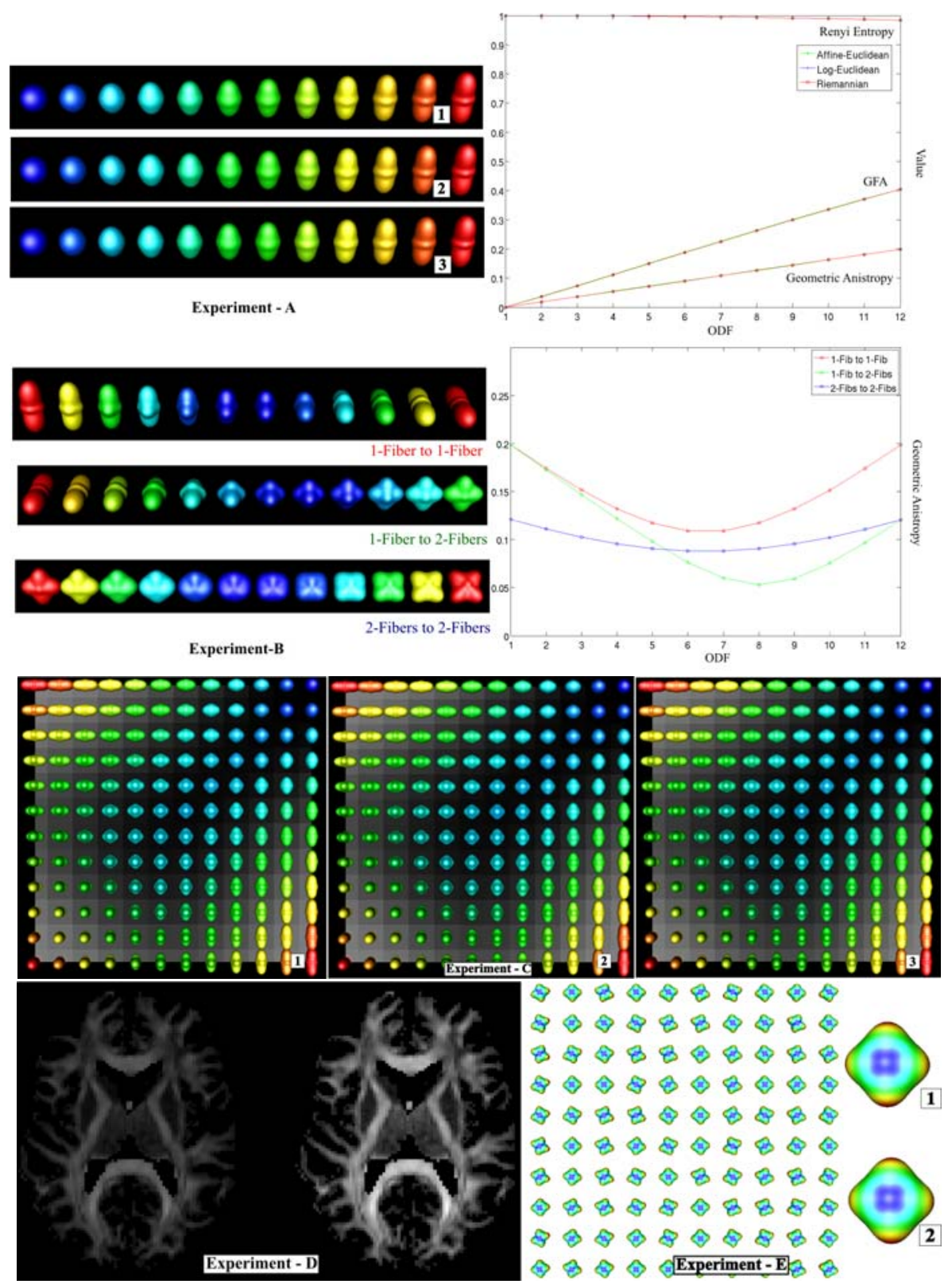

Fig. 2. Exp-A: Interpolation from Isotropic to 1-fiber using (1) AE, (2) LE, (3) RM metrics. Riemannian (RM) GA is linear as expected. Exp-B: Various RM geodesics with corresponding GA plotted. Exp-C: 2D Lagrange interpolation (given 4 ODFs in the 4 corners) using (1) AE, (2) LE, (3) RM metrics. Background is GA. Exp-D: GA (left) and GFA (right) from clinical real data. ODFs estimated using [8] Exp-E: (1) LE \& (2) RM mean from a field of ODFs. Results and computation time of the metrics are comparable. For mean computation, LE has a closed form. 
robust. The weighted Frechet mean exists uniquely. Moreover, we present the AE and the LE frameworks so that the computation is simplified further and we have closed forms. A Lagrange interpolation method was proposed as an application. The results validate our framework. Last but not least, this framework can be used for any kind of PDF under orthonormal basis representation, which is part of our future plans.

\section{References}

1. Basser, P.J., Mattiello, J., LeBihan, D.: Mr. diffusion tensor spectroscropy and imaging. Biophysical Journal 66, 259-267 (1994)

2. Pennec, X., Fillard, P., Ayache, N.: A riemannian framework for tensor computing. International Journal of Computer Vision 66, 41-66 (2006)

3. Lenglet, C., Rousson, M., Deriche, R.: Statistics on the manifold of multivariate normal distributions theory and application to diffusion tensor mri processingy. Journal of Mathematical Imaging and Vision 25(3), 423 (2006)

4. Arsigny, V., Fillard, P., Pennec, X., Ayache, N.: Log-euclidean metrics for fast and simple calculus on diffusion tensors. Magnetic Resonance in Medicine 56, 411-421 (2006)

5. Fletcher, P.T.: Statistical Variability in Nonlinear Spaces Application to Shape Analysis and DT-MRI. PhD thesis, University of North Carolina (2004)

6. Tuch, D.S.: Q-ball imaging. Magnetic Resonance in Medicine 52, 1358-1372 (2004)

7. Descoteaux, M., Angelino, E., Fitzgibbons, S., Deriche, R.: Apparent diffusion coefficients from high angular resolution diffusion images: Estimation and application. Magnetic Resonance in Medicine 56, 395-410 (2006)

8. Descoteaux, M., Angelino, E., Fitzgibbons, S., Deriche, R.: Regularized, fast and robust analytical q-ball imaging. Magnetic Resonance in Medicine 58, 497-510 (2007)

9. Kezele, I., Descoteaux, M., Poupon, C., Abrial, P., Poupon, F., Mangin, J.F.: Multiresolution decomposition of hardi and odf profiles using spherical wavelets. In: MICCAI (2008)

10. Descoteaux, M., Deriche, R.: High angular resolution diffusion mri segmentation using region-based statistical surface evolution. Journal of Mathematical Imaging in Vision special issue on Mathematics in Image Analysis, 305-317 (2008)

11. McGraw, T., Vemuri, B.C., Yezierski, R., Mareci, T.H.: Segmentation of high angular resolution diffusion MRI modeled as a field of von mises-fisher mixtures. In: Leonardis, A., Bischof, H., Pinz, A. (eds.) ECCV 2006. LNCS, vol. 3953, pp. 463-475. Springer, Heidelberg (2006)

12. Rathi, Y., Michailovich, O., Shenton, M.E., Bouix, S.: Directional functions for orientation distribution estimation. Medical Image Analysis, 432-444 (2009)

13. Amari, S., Nagaoka, H.: Methods of Information Geometry. American Mathematical Society, USA (2000)

14. Buss, S.R., Fillmore, J.P.: Spherical averages and applications to spherical splines and interpolation. ACM Transactions on Graphics 20, 95-126 (2001)

15. Srivastava, A., Jermyn, I.: Riemannian analysis of probability density functions with applications in vision. In: CVPR (2007)

16. Dryden, I.L., Mardia, K.V.: Statistical Shape Analysis. John Wiley, Chichester (1998)

17. Descoteaux, M.: High Angular Resolution Diffusion MRI: from Local Estimation to Segmentation and Tractograpghy. PhD thesis, INRIA Sophia Antipolis (2008) 